logical report of two cases with a contribution to pathogenesis and

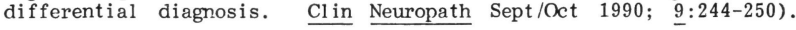

COMNENT. Cerebral hemiatrophy with homolateral hemihypertrophy of the skull and sinuses was described by Dyke OG, Davidoff L, and Masson C. (Arch Neurol and Psychiat 1933; 29:412)

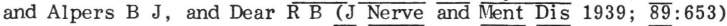
distinguished the primary and secondary groups of cases. The significance of convulsions in the pathogenesis of cerebral hemiatrophy is emphasized by many authors - the "hemiconvulsion hemiplegia - epilepsy syndrome." Permanent hemiparesis as a direct result of a febrile convulsion is a rare complication occurring in less than $0.2 \%$ (Millichap J. G. Febrile Convulsions, Macmillan, New York, 1968).

\title{
CHARGE SYNDROME
}

A 12 month old male infant with the CHARGE syndrome (Coloboma, Heart defect, Atresia of the choana, Retarded growth and development, Genital hypoplasia, Ear anomalies or deafness) is reported from the Departments of Pediatrics and Neurology, Loyola University, Chicago. The infant was admitted with repeated cyanotic episodes and generalized seizures. CT revealed agenesis of the cerebellar vermis and a large fourth ventricular cyst. Brain stem auditory evoked responses showed dysfunction of the distal VIII nerve (Menenzes M, Coker S. B. CHARGE and Joubert's Syndromes, Are They a Single Disorder? Pediatr Neurol Nov/Dec $1990 ; \underline{6}: 428-430)$.

COMMENT. This patient also had Joubert's syndrome (agenesis of the cerebellar vermis, respiratory irregularities, ocular colobomas). Chromosames were normal. The authors believe that these two syndromes are a single disorder with an overlap of characteristic features.

Various associated abnormalities of brain development may be detected by studies of evoked potentials (Coupland S G, Sarnat $\mathrm{H}$ B Visual and auditory evoked potential correlates of cerebral malformations, Brain Dey $1990 ; 12: 466-72)$. Auditory brain stem responses were abnormal in cases of holoprosencephaly, lissencephaly, pachygyria and generalized megalencephaly. Visual evoked potentials were normal in collosal agenesis, holoprosencephaly and colpocephaly; they were abnormal in septal optic dysplasia and opt ic nerve hypoplasia.

\section{MUSCLE DISORDERS}

MYOPATHY WITH COMPLEX I DEFICIENCY: RIBOFLAVIN CARNITINE THERAPY

A six year old boy with progressive myopathy and motor neuropathy associated with complex I deficiency is reported from the Institutes of 
Neurology, Pediatrics and Cell Biology, St. Radboud University Hospital, Nijmegen, Netherlands. There was a three month history of weakness in the legs, sudden falling, inability to run and difficulty in climbing stairs. Within two months he developed weakness in his hands, a wobbling gait and a positive Gowers' sign. Muscle tone was impaired and proximal muscles were wasted. Biochemical studies on muscle tissues showed a defect of NADH dehydrogenase (camplex I). Dramatic improvement followed oral treatment with L-carnitine $2 \mathrm{~g}$ daily and riboflavin $9 \mathrm{mg}$ daily. Seven months later the complex I activity was normal, Gowers' sign was negative, deep tendon reflexes had returned and muscle weakness was limited mainly to the peroneal muscles. Nine further cases of a pure myopathy associated with complex I deficiency are reviewed (Bernsen PLJA et al. Successful treatment of pure myopathy, associated with complex I deficiency, with riboflavin and carnitine. Arch Neurol March 1991; 48:334-338).

COMMENT. The present case report of a mitochondrial myopathy differed from nine other reports. There was no history of excessive fatiguability or exercise intolerance, the serum lactate concentration was normal, and the muscle biopsy did not show ragged red fibers. The dramatic improvement with riboflavin and carnitine and the return to normal of complex I activity were unusual findings. The authors stress the clinical, biochemical and morphological heterogeneity in complex I deficiency. Biochemical studies of mitochondrial metabolism in the muscle are advisable in children with progressive weakness and exercise intolerance when a precise diagnosis is unclear.

FATAL AND RENIGN CONGENITAL MYOPATHIES: DIFFEPENTIAL DIAGNOSIS

Nuscle biopsies from four infants with fatal myopathy and four with benign myopathy were examined using biochemical, histochemical and immunohistochemical techniques in the Departments of Neurology, Pathology and Genetics, Columbia University, New York; Universita Cattolica del Sacro Cuore, Rome, Italy; and Fachbereich Chemie, Philipps-Universitat, Marburg, Germany. At early stages the clinical picture failed to provide clues for differential diagnosis; both fatal and benign myopathies presented with hypotonia, generalized weakness, lactic acidosis, failure to thrive and severe respiratory insufficiency, often requiring assisted ventilation. Patients with benign myopathy gain strength and show an increase in the number of fibers with COX (cytochrame c oxidase) deficient activity. In contrast, patients with fatal myopathy die before one year of age and muscle histochemistry shows no increase in the number of fibers with $\operatorname{COX}$ activity. The subunit composition of $\mathrm{COX}$ was studied directly on frozen muscle sections using immunologic probes. The fatal type was characterized by absence of the nuclear DNA-encoded subunit VIIa,b of COX while in the benign myopathy both VIIa,b and the mitochondrial DNA-encoded subunit II were absent. From a practical standpoint, immunohistochemistry of COX-II should suffice for differential diagnosis because this subunit was present in the fatal myopathy but absent in the early stages of the benign myopathy (Tritscler HJ, DiMlauro $S$ et al Differential diagnosis 